\title{
Diferencias en afrontamiento, bienestar y satisfacción entre desempleados y empleados en cuanto al género
}

Carolina Delgado García, Claudia Espejo Artacho, Jacqueline Moreno Zurita y Francisco Javier Domínguez Martos. Universidad de Granada

Recepción: 12 de mayo de 2015 | Revisión: 17 de junio de 2015 | Aceptación/Publicación: 17 de julio de 2015

Correspondencia: ivalor@ugr.es

Citar: Delgado, C., Espejo, C., Moreno, J. y Dominguez, F. (2015). Diferencias en afrontamiento, bienestar y satisfaccion entre desempleados y empleados en cuanto al género. ReiDCrea, 4: 85-93. [http://hdl.handle.net/10481/37008]

\begin{abstract}
Resumen: El objetivo de la presente investigación ha sido analizar el grado de satisfacción laboral y el afrontamiento al desempleo dependiendo del género, al igual que estudiar la repercusión del paro en el bienestar psicológico de los sujetos, esperando como hipótesis de partida que los hombres se verán más influenciados por el desempleo y que tengan una mayor satisfacción laboral. La novedad del presente artículo se debe a la situación de crisis económica en la que España está sumergida, para saber cómo los desempleados afrontan la situación a la que se enfrentan, y cómo de satisfechos están los afortunados que actualmente están trabajando. Al contrario de lo esperado, no se han encontrado diferencias significativas entre hombres y mujeres, mientras que sí se han encontrado diferencias significativas entre empleados y desempleados, donde los empleados muestran mayor satisfacción laboral, al igual que se han encontrado diferencias significativas en las estrategias de afrontamiento utilizadas.
\end{abstract}

Palabras clave: satisfacción laboral, bienestar laboral, estrategias de afrontamiento, género.

\section{Introducción}

La crisis económica ha tenido un gran impacto en toda la Unión Europea, pero en España sus consecuencias han sido devastadoras en el mercado laboral, llegando incluso a alcanzar la cifra de más de cuatro millones de parados. El aumento del desempleo se caracteriza por cambios estructurales en el mercado de trabajo de todos los países industrializados (Escribà-Agüir y Fons-Martinez, 2014).

España ha pasado a ser el país de la Unión Europea con peores indicadores de empleo. La situación es peor en la población joven, de la cual en 2012 una de cada dos personas estaba en situación de desempleo, con una tasa algo superior en los hombres $(54,4 \%$ frente a $51,8 \%$ en las mujeres) (Escribà-Agüir, y Fons-Martinez, 2014). Todo ello ha comportado reformas en las políticas del mercado de trabajo (regulación laboral y de las relaciones industriales) y del estado de bienestar (políticas sociales de empleo). Como resultado, también cabe esperar que se produzca un empeoramiento de las condiciones de empleo (trabajo no estable, trabajo a tiempo parcial, trabajo informal, paro), es decir, un empeoramiento de las condiciones de trabajo de tipo físico, y de la organización del trabajo (ambiente psicosocial, jerarquías y relaciones de poder, participación de los trabajadores y trabajadoras, discriminación social y laboral, etc) (Escribà-Agüir, y Fons-Martinez, 2014).

El desempleo también influye en la satisfacción laboral de los trabajadores, dando lugar a empleos poco remunerados, que se convierte en la esperanza de personas que trabajan un gran número de horas por un salario bajo con el fin de mantener a su familia y conservar su hogar. Mañas et al. (2007) apuntó que el compromiso con la organización dependía de la satisfacción laboral, en otras palabras, los empleados decidirán comprometerse con la organización si se encuentran contentos en la misma.

La satisfacción laboral depende de varios factores, como la variable sexo, en un estudio de Sloane y Williams (2000), las mujeres tenían más satisfacción laboral que los hombres debido a que su situación en el mercado laboral es más insegura que los hombres lo que les daba menos expectativas. Respecto a la edad, se encontró que al 
aumentar esta, aumentaba la satisfacción laboral ya que influía en su percepción objetiva de su situación laboral (Clark, Oswald y Warr, 1996).

La antigüedad en la organización era un predictor positivo de la satisfacción laboral. También hay otras variables como la relación con los compañeros, el reconocimiento por parte de la dirección, así como el equilibrio con la vida privada. Por último se encuentra el ambiente de trabajo: ruido, iluminación, diseño del espacio de trabajo, etc. (O’Neill, 1992).

En cuanto al desempleo, la falta de este no sólo constituye un fracaso del sistema social, sino que también constituye una vía de privaciones materiales, afectación subjetiva y degradación social para quienes padecen sus consecuencias. No disponer de un trabajo constituye una fuente de deterioro del sentido de identidad a nivel de género (Burin et al., 2004). El desempleo produce el deterioro del estatus social y esto puede afectar a la autoestima del desempleado. Si es duradero, puede generar incluso distintos problemas de salud como los trastornos del estado de ánimo, la depresión, e incluso trastornos de personalidad, produciendo un sentimiento de fracaso, inferioridad, inutilidad, dependencia, pérdida de autoestima, vergüenza, ira, tristeza, culpabilidad, frustración y sintomatología depresiva, entre otros muchos más (Rita y Salvia, 2003).

Las estrategias de afrontamiento son los procesos concretos que se utilizan en cada contexto y pueden ser altamente cambiantes dependiendo de las condiciones desencadenantes (Fernández-Abascal, 1997). Hay diferencias individuales ante la elección estrategias de afrontamiento de este tipo de problemas que pueden ser favorables o no favorables para la salud. Estrategias como "evaluar las dificultades con las que se enfrentan, sin caer en dramatismos" o "buscar el apoyo social de familia y allegados con los que desahogarse", son estrategias favorecedoras para la salud psicológica del desempleado (Buendía, 2012). Existen diferencias significativas entre sexos en la utilización de estrategias de afrontamiento, por ejemplo en la estrategia de control emocional los hombres puntúan más alto que las mujeres, en apoyo social al problema los hombres puntúan más bajo que las mujeres al igual que en la expresión emocional (Martín y Jiménez, 2000).

Debido a la gravedad de la crisis, es interesante saber las diferentes maneras de afrontamiento del desempleo en hombres y mujeres, estudiando la repercusión del paro en el bienestar psicológico de los sujetos, entendiendo este como la ausencia de indicadores negativos, que mantiene una correlación positiva con la apreciación de la vida y su mantenimiento a lo largo del tiempo, que depende del equilibrio entre expectativas y logros conseguidos (Diener, 1994). Además de esto, se quiere saber el grado de satisfacción laboral dependiendo del género. Por satisfacción laboral entendemos el sentimiento de agrado que experimenta un sujeto por el hecho de realizar un trabajo que le interesa, dentro de una organización que le resulta atractiva y por el que recibe una serie de compensaciones psico-socio-económicas acordes con sus expectativas (Bravo, Peiró y Rodríguez, 1996).

Se predice que tras realizar el estudio, los resultados van a indicar que a los hombres les afectan más el desempleo que a las mujeres, tanto psicológicamente como en el ámbito social. También se piensa que los hombres tendrán más satisfacción laboral, debido a los ingresos, que las mujeres. 


\section{Método}

\section{Participantes}

La muestra seleccionada fueron 60 personas de las cuales la mitad fueron mujeres y la otra mitad hombres. A su vez, la muestra se dividía en 30 desempleados y 30 empleados. Al final la muestra quedó 15 mujeres desempleadas, 15 mujeres empleadas, 15 hombres desempleados y 15 hombres empleados.

La media de edad de los participantes estaba comprendida entre 20 y 59 años $(M=32$ $D T=12,4$ ), todos de nacionalidad española con diferentes provincias (Córdoba, Málaga y Granada).

Los sujetos se clasifican en empleados y desempleados. Dentro del grupo de los empleados encontramos distintas modalidades: autónomos (3), contrato fijo (14), contrato temporal (9), y contrato parcial (4), siendo el grupo de contrato fijo el más numeroso. Dentro del grupo de desempleados, el tiempo sin trabajar estaba comprendido entre 0 y 96 meses $(M=18,67 D T=33,22)$.

También se ha medido el nivel de estudios, clasificados en: sin estudios (0), estudios primarios (4), estudios secundarios (13), bachillerato (20) y Grado o Licenciatura (23), siendo el más numeroso Grado o Licenciatura.

Finalmente, también se ha medido el estado civil en distintas modalidades: soltero/soltera (34), casado/ casada (19), viudo/viuda (1), conviviendo (3), y divorciado/divorciada (3), siendo el más numeroso la modalidad soltero/ soltera.

\section{Diseño}

Se ha llevado a cabo una investigación no experimental de tipo correlacional, pues el objetivo del estudio es describir la correlación entre varias variables (afrontamiento, bienestar laboral y satisfacción laboral).

\section{Instrumentos}

Para llevar a cabo esta investigación se han usado tres cuestionarios. El primer cuestionario utilizado es el Inventario de Estrategias de Afrontamiento (Tobin, Holroyd, Reynolds y Kigal, 1989; adaptación por Cano, Rodríguez y García, 2006). El cuestionario se compone de 40 ítems, los ítems se puntúan desde 0 a 4,0 equivaldría a "En absoluto" y 4 a "Totalmente". El cuestionario se subdivide en 8 subescalas: Resolución de Problemas (REP), Autocrítica (AUC), Expresión Emocional (EEM), Pensamiento desiderativo (PSD), Apoyo Social (APS), Reestructuración cognitiva (REC), Evitación de Problemas (EVP) y Retirada Social (RES). La puntuación final de cada escala se calcula sumando la puntuación de cada ítem relacionado con la escala habiendo 5 ítems por escala. En relación a la fiabilidad de este cuestionario en esta investigación, se realizó el alfa de Cronbach con un resultado obtenido de 0,91. .

El segundo instrumento utilizado fue el Cuestionario de Satisfacción Laboral S10/12 (Melía y Peiró, 1998). Consta de 12 ítems, los cuales se puntúan en una escala del 1 a 7 , siendo 1 "muy insatisfecho" y 7 "muy satisfecho", siempre teniendo en cuenta el ítem del cual se pregunta. La puntuación final se realiza haciendo la media de la puntuación directa de cada ítem cuya puntuación máxima sería de 7 . La forma de los ítems se expresa de la siguiente manera "La proximidad y frecuencia con que es supervisado" y "Los objetivos, metas y tasas de producción que debe alcanzar", entre 
otros. En relación a la fiabilidad de este cuestionario en esta investigación, se realizó el alfa de Cronbach con un resultado obtenido de 0,91.

La escala que utilizamos provenía de la Escala de Bienestar Psicológico (CánovasSánchez, 1988), el cual consta de 4 subescalas, nosotros hemos usado la escala de bienestar laboral. La escala consta de 10 ítems, estos se puntúan en una escala de 1 a 5 , siendo 1 "de acuerdo" con la afirmación presentada y 5 "desacuerdo" con la afirmación. La escala de bienestar material tiene como puntuación máxima 50 , esta puntuación se obtiene sumando las puntuaciones directas de todos los ítems. Los ítems están escrito de forma "Tengo lo necesario para vivir", "Mi situación es relativamente prospera". En relación a la fiabilidad de estas subescalas en esta investigación, se realizó el alfa de Cronbach con un resultado obtenido de 0,80.

Para saber la fiabilidad total del cuadernillo que se han pasado a los sujetos se procedió a realizar el alfa de Cronbach, obteniendo como resultado realizó el alfa de Cronbach con un resultado 0,77 .

\section{Procedimiento}

Los participantes del estudio fueron seleccionados debido a la cercanía a los experimentadores, siendo padres, familiares y otras personas cercanas, además se buscó más participantes en tiendas, puestos de trabajo y en la calle. Se les explicó que estábamos realizando una investigación para la asignatura de Psicología del Trabajo y de las Organizaciones de la Facultad de Granada, la hipótesis de estudio, que leyesen el consentimiento informado y lo firmasen para poder continuar. Tras realizar esto se administraron los cuestionarios y se les explicó cómo debían rellenarlo, los participantes lo rellenaron en su tiempo libre y en un lugar tranquilo para no interferir con su trabajo y su vida diaria.

\section{Resultados}

El análisis de datos realizado en este apartado se realizó con el programa estadístico SPSS 22.0 para Windows. El objetivo último fue demostrar, tras haber cuantificado las variables y haber utilizado el procedimiento estadístico adecuado, si existía una relación cuantitativamente significativa entre los datos obtenidos en afrontamiento, satisfacción laboral y bienestar laboral, a nivel general.

Se procedió a constatar si existían diferencias significativas entre empleados y desempleados en la satisfacción laboral, en el bienestar psicológico y en las distintas subescalas de afrontamiento. Para ello, se utilizó una prueba $t$ de student para muestras independientes, que reflejó diferencias significativas entre empleados y desempleados en satisfacción laboral con $t(55,29)=.002, p<.05$; en autocrítica (AUC) con $t(57,54)=.046, p<.05$ y resolución de problemas $(\operatorname{REP})$ con $t(57,51)=.049, p<.05$. Por lo que se interpreta que, los empleados tienen mayor satisfacción en su trabajo, solucionan mejor los problemas a los que se enfrentan y se autocritican más que los desempleados. Todos estos resultados se reflejan en la siguiente gráfica: 
Gráfica 1. Diferencias entre empleados y desempleados en las diferentes variables de interés.

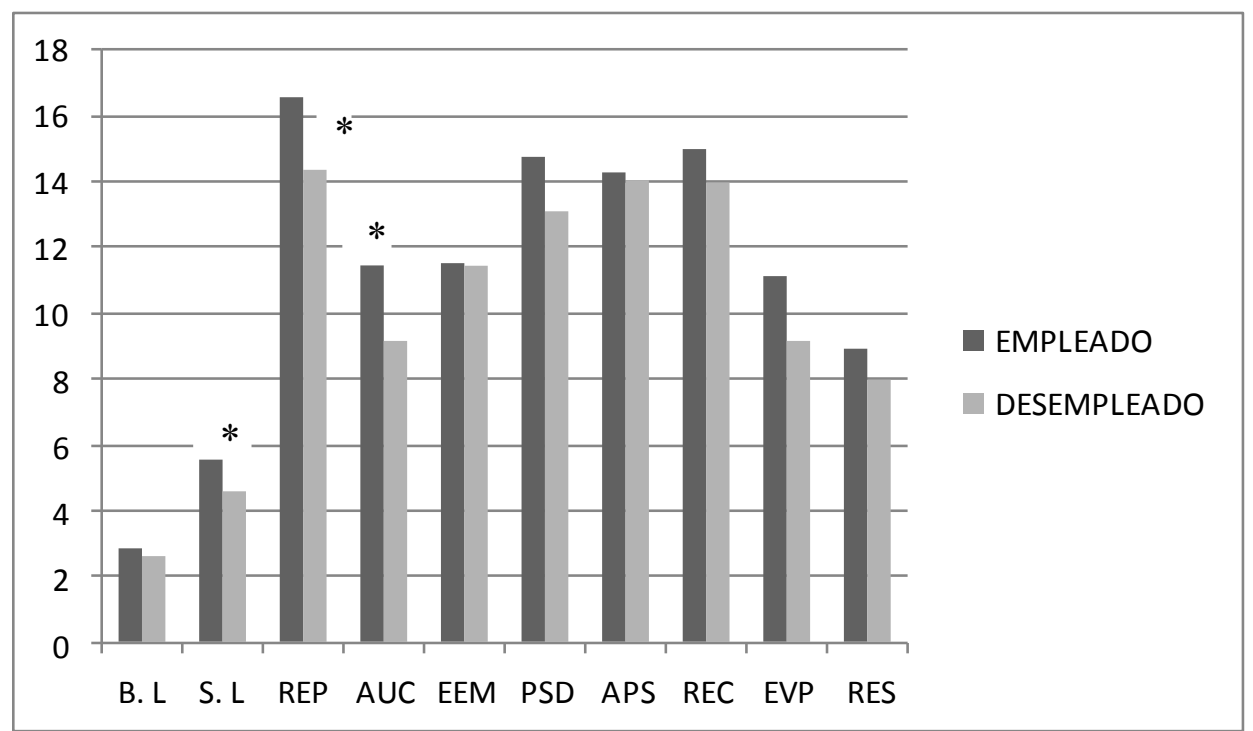

Nota: * p < .05; B.L: Bienestar laboral; REP: resolución de problemas; EEM: expresión emocional; S.L: Satisfacción laboral; AUC: autocrítica; PSD: pensamiento desiderativo; APS: apoyo social; REC: reestructuración cognitiva; EVP: evitación de problemas; RES: retirada social.

En cuanto a las diferencias entre hombres y mujeres, vemos que existen diferencias en pensamiento desiderativo (PSD), donde las mujeres presentan unos niveles ligeramente mayores que los hombres, es decir tienen mayores pensamientos de deseo sobre algunas situaciones que ellos, pero no llega a ser un diferencia significativa $(p>.05)$. En el resto de las variables evaluadas tampoco hay diferencias significativas entre hombres y mujeres.

Gráfica 2. Diferencias entre hombres y mujeres en las diferentes variables de interés.

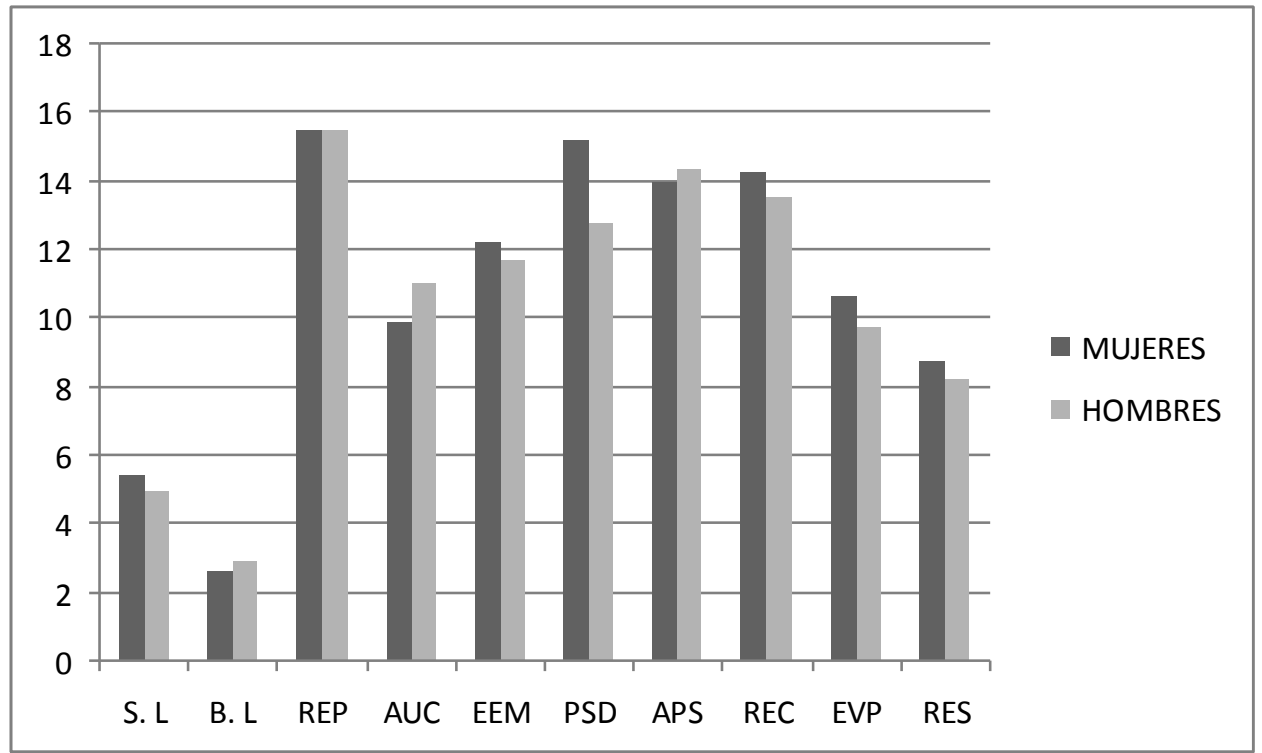

Nota: * $\mathrm{p}<.05$; B.L: Bienestar laboral; REP: resolución de problemas; EEM: expresión emocional; S.L: Satisfacción laboral; AUC: autocrítica; PSD: pensamiento desiderativo; APS: apoyo social; REC: reestructuración cognitiva; EVP: evitación de problemas; RES: retirada social. 
En última instancia, se procedió a comparar las correlaciones (tanto positivas (cuando aumenta una variable aumenta la otra) como negativas (cuando aumenta una variable disminuye la otra)) de las diferentes variables de nuestro estudio (satisfacción laboral, bienestar material y afrontamiento junto con sus correspondientes subescalas), entre hombres y mujeres.

Tal y como se observa en la tabla, se han encontrado correlaciones positivas en mujeres en las variables resolución de problemas (REC), expresión emocional (EEM), apoyo social (APS), reestructuración cognitiva (REC) y evitación de problemas (EVP), con respecto a la satisfacción laboral. En cuanto a la retirada social, existen correlaciones positivas con la autocrítica; aunque también existen correlaciones negativas con resolución de problemas y expresión emocional. La resolución de problemas (REP) correlaciona positivamente con expresión emocional (EEM), reestructuración cognitiva (REC), apoyo social (APS) y pensamientos desiderativos (PSD). La expresión emocional correlaciona positivamente con apoyo social (APS), reestructuración cognitiva (REC) y con evitación de problemas (EVP). Los pensamientos desiderativos (PSD) y apoyo social (APS), ambos correlacionan positivamente con reestructuración cognitiva (REC). Por último, la reestructuración cognitiva (REC) correlaciona positivamente con evitación de problemas (EVP).

Con respecto a los hombres, existen correlaciones positivas entre autocrítica (AUC) y retirada social (RES). La expresión emocional (EEM), correlaciona positivamente con la retirada social (RES) y autocrítica (AUC). Los pensamientos desiderativos (PSD), correlaciona positivamente con retirada social (RES), autocrítica (AUC) y expresión emocional (EEM). El apoyo social (APS) correlaciona positivamente con la autocrítica (AUC), con expresión emocional (EEM) y el pensamiento desiderativo (PSD). La reestructuración cognitiva (REC) correlaciona positivamente con resolución de problemas (REP), retirada social (RES) y pensamientos desiderativos (PSD). Finalmente, la evitación de problemas (EVP) correlaciona positivamente con la retirada social (RES), la autocrítica (AUC), la expresión emocional (EEM) y el pensamiento desiderativo (PSD). Los datos se encuentran presentes en la siguiente tabla:

\begin{tabular}{|c|c|c|c|c|c|c|c|c|c|c|}
\hline \multicolumn{11}{|c|}{$\begin{array}{c}\text { Tabla } 1 \\
\text { Correlaciones entre variables } \\
\end{array}$} \\
\hline & SL & $\mathrm{BL}$ & RES & REP & AUC & EEM & PSD & APS & REC & EVP \\
\hline SL & -- & -.23 & -.11 & $.51^{* *}$ & .11 & $.38^{*}$ & .35 & $.38^{*}$ & $.46^{\star \star}$ & $.37^{*}$ \\
\hline $\mathrm{BL}$ & .13 & -- & .04 & -.05 & .18 & -.23 & .00 & -.19 & -.19 & -.10 \\
\hline RES & .06 & .21 & -- & $-.41^{*}$ & $.57^{\star *}$ & $-.51^{* *}$ & .08 & $-.61^{* *}$ & -.33 & .06 \\
\hline REP & .36 & .01 & .02 & -- & -.18 & $.70^{\star \star}$ & $.58^{* *}$ & $.70^{\star *}$ & $.82^{\star \star}$ & .40 \\
\hline AUC & -.15 & .15 & $.61^{* *}$ & .06 & -- & -.30 & .19 & -.21 & -.08 & .19 \\
\hline EEM & -.17 & .07 & $.39^{*}$ & -.04 & $.63^{* *}$ & -- & .32 & $.88^{* *}$ & $.67^{\star \star}$ & $.47^{\star \star}$ \\
\hline PSD & .03 & .07 & $.50^{\star *}$ & .05 & $.75^{\star *}$ & $.62^{\star \star}$ & -- & .24 & $.42^{*}$ & .25 \\
\hline APS & -.03 & .11 & .21 & .02 & $.64^{* *}$ & $.66^{\star *}$ & $.56^{* *}$ & -- & $.72^{* *}$ & .31 \\
\hline REC & .16 & .04 & $.44^{*}$ & $.64^{\star *}$ & .34 & .35 & $.47^{* *}$ & .32 & -- & $.41^{*}$ \\
\hline EVP & -.12 & .25 & $.77^{* *}$ & -.00 & $.58^{\star *}$ & $.51^{* *}$ & $.50^{* *}$ & .25 & .52 & -- \\
\hline \multicolumn{11}{|c|}{$\begin{array}{l}\text { Nota: }{ }^{* *} \mathrm{p}<.05{ }^{*} \mathrm{p}<.01 \\
\text { En la diagonal superior se encuentran los datos correspondientes a las mujeres. } \\
\text { En la diagonal inferior se encuentran los datos correspondientes a los hombres. } \\
\text { B.L: Bienestar laboral; REP: resolución de problemas; EEM: expresión emocional; S.L: Satisfacción laboral; AUC } \\
\text { autocrítica; PSD: pensamiento desiderativo; APS: apoyo social; REC: reestructuración cognitiva; EVP: evitación de } \\
\text { problemas; RES: retirada social. }\end{array}$} \\
\hline
\end{tabular}




\section{Discusión}

Debido a que la literatura que expresa las grandes dificultades que se encuentran en el ámbito laboral y a las consecuencias negativas que conlleva el desempleo está proliferando, y debido a la época de crisis a la que nos enfrentamos; se decidió estudiar las diferencias de afrontamiento entre hombres y mujeres con respecto al desempleo y las diferencias entre los mismos respecto a la satisfacción y bienestar laborales (Buendía, 2010).

Los resultados obtenidos permiten concluir que sí existen diferencias significativas en la satisfacción laboral entre empleados y desempleados $(t(55,29)=.002, p<.05)$ al igual que en afrontamiento, destacando diferencias significativas en la estrategia de resolución de problemas con $t(57,51)=.049, p<.05$, y autocrítica con $t(57,54)=.046$, $p<.05$. Sin embargo, en hombres y mujeres se han encontrado diferencias, pero éstas no llegan a ser significativas, concretamente hay ligeras diferencias en la subescala de afrontamiento" "pensamientos desiderativos" y "satisfacción laboral".

Hay que tener en cuenta que también puede haber diferencias entre culturas, por ejemplo, los hombres suecos no se ven más afectados, en cuanto a consecuencias sanitarias, por el desempleo que las mujeres (Hammarström, Gustafsson, Strandh, Virtanen y Janlert, 2011).

Aunque la hipótesis de partida era encontrar mayor afectación por parte de los hombres ante el desempleo y ante la satisfacción laboral, no se han encontrado diferencias significativas para apoyar dicha hipótesis, sin embargo, como se ha comentado anteriormente, sí hay ciertas diferencias significativas entre empleados y desempleados (Gálvez y Rodríguez, 2011).

En cuanto a las limitaciones, destaca el reducido tamaño de la muestra (60 sujetos). Es posible que una muestra de mayor tamaño hubiera facilitado el encontrar diferencias claramente significativas tanto en hombres y mujeres, como en empleados y desempleados. Además, puede ser que se encontraran mayor número de diferencias de las que este estudio ha destacado.

La satisfacción es una variable muy importante independientemente del género en el ámbito laboral, dado que puede influir en la realización del trabajo, en la salud (reduciendo el estrés o depresión) o aumentando el rendimiento. Igual de importante es el bienestar laboral, tanto en las relaciones con los compañeros de trabajo, como en las condiciones del lugar de trabajo (temperatura óptima, comodidad, etc.)

\section{Bibliografía}

Augusto, J.M. y Martínez, R. (1998). Afrontamiento al estrés y salud: panorama actual de la investigación. Boletín de Psicología, 58, 31-48.

Bòria-Reverter, S., Crespi-Vallbona, M., y Mascarilla-Miró, O. (2012). Variables determinantes de la satisfacción laboral en España. Cuadernos de economía, 35, 9-16.

Borzaga, C., y Tortia, E. (2006). Worker Motivations, Job Satisfaction, and Loyalty in Public and Nonprofit Social Services. Nonprofit and Voluntary Sector Quarterly, 35 (2), 225-248. doi:10.1177/0899764006287207.

Bravo, Ma.J., Peiró, J.M. y Rodríguez, I. (1996). Satisfacción laboral. Tratado de Psicología del Trabajo, 1, (pp.347394).

Buendía, J. (2010). El impacto psicológico del desempleo. Editum. 
Burin et al. (2004): Género, psicoanálisis, subjetividad. Paidós, Buenos Aires.

Camacho, B., García, D., León, E., Mateos, A., y Peña, M. (2013). Relación entre las dimensiones del síndrome de Burnout y los factores de satisfacción laboral. ReiDoCrea, 2, 33-40. http://digibug.ugr.es/handle/10481/27614

Clark, A. E., Oswald, A. J. y Warr, P. B. (1996). Is job satisfaction U-shaped in age? Journal of Occupational and Organizational Psychology, 69, 57-81.

Diener, E. (1994). El bienestar subjetivo. Intervención Psicosocial, 3, 67-113.

Escribà-Agüir, V. y Fons-Martinez, J. (2014). Crisis económica y condiciones de empleo: diferencias de género y respuesta de las políticas sociales de empleo. Informe SESPAS 2014. Gaceta Sanitaria, 28(1), 37-43.

Fernández-Abascal, E. G., Palmero,F., Chóliz, M., y Martínez, F. (1997). Cuaderno de Prácticas de Motivación y Emoción. Madrid. Pirámide.

Figueroa, M. I., Contini, N., Lancuza, A. B., Levín, M. y Estévez Suedan, A. (2005). Las estrategias de afrontamiento y su relación con el nivel de Bienestar psicológico. Anales de Psicología, 21(1), 66-72.

Gálvez, L. y Rodríguez, P. (2011). La desigualdad de género en las crisis económicas, Investigaciones Feministas, 2 , 113-132.

González, F., Sánchez, S. y López-Guzmán, T. (2011). Satisfacción laboral como factor crítico para la calidad: el caso del sector hostelero de la provincia de Córdoba-España. Estudios y Perspectivas en Turismo, 20, 1047-1068.

Goodin, R. E. (2003). Democratic Accountability: The Distinctiveness of the Third Sector. European Journal of Sociology, 44 (3), 359-396. doi:10.1017/S0003975603001322.

Hammarström, A., Gustafsson, P.E. Strandh M., Virtanen, P., Janlert, U. (2011). It's no surprise! Men are not hit more than women by the health consequences of unemployment in the Northern Swedish Cohort. Scandinavian Journal of Public Health, 39, 187.

Judge, T.A., Piccolo, R.F., Podsakoff, N.T., Shaw, J.C. y Rich, B.L. (2010). The Relationship between Pay and Job Satisfaction: A Meta-Analysis of the Literature. Journal of Vocational Behavior, 77, $157-167$. doi:10.1016/j.jvb.2010.04.002.

Lee y Sabharwal (2014). Education-Job Match, Salary, and Job Satisfaction across the Public, Non-Profit, and ForProfit Sectors: Survey of recent college graduates, Public Management Review, 1-25.

Lee, Y., y Wilkins, V. M. (2011). More Similarities or More Differences? Comparing Public and Nonprofit Managers' Job Motivations. Public Administration Review, 71(1), 45-56. doi:10.1111/j.1540-6210.2010.02305.x.

Liu, B., y Tang, T. (2011). Does the Love of Money Moderate the Relationship between Public Service Motivation and Job Satisfaction? The Case of Chinese Professionals in the Public Sector. Public Administration Review, 71(5), 718-727

Liu, X., Thomas, S. y Zhang, L. (2010). College Quality, Earnings, and Job Satisfaction: Evidence from Recent College Graduates. Journal of Labor Research, 31 (2), 183-201.

Lyons, S., Duxbury, L. y Higgins, C. A. (2006). A Comparison of the Values and Commitment of Private Sector, Public Sector, and Parapublic Sector Employees. Public Administration Review, 66 (4), 605-618. doi:10.1111/j.15406210.2006.00620.x

Mañas, M. A, Salvador, C., Boada, J., González, E., y Agulló, E. (2007). La satisfacción y el bienestar psicológico como antecedentes del compromiso organizacional. Psicothema, 19 (3), 395-400.

Martín, D.M. y Jiménez, S.M. (2000). Estudio sobre la escala de estilos y estrategias de afrontamiento. Revista electrónica de motivación y emoción, 3.

Moreno, M.P., Ríos, M.L., Canto, J.M., San Martín, J. y Perles, F., (2010). Satisfacción laboral y burnout en trabajos poco cualificados: diferencias entre sexos en población inmigrante. Revista de Psicología del Trabajo y de las Organizaciones, 26 (3), 255-265.

Mumford, K., y Smith, P. (2014). Peer salaries and gender differences in job satisfaction in the workplace. The Manchester School. doi: 10.1111/manc.12060 
Rita, B.R. y Salvia, A. (2003). Descomposición social del malestar subjetivo y de las capacidades de afrontamiento en un contexto de crisis y desempleo. Universidad de Buenos Aires.

Slone, P. y Williams, H. (2000). Job satisfaction, comparison earnings and gender. Labour, 14, 473-502. 